\title{
A Crystallographic, Spectroscopic and Computational Investigation of Carbonyl and Oxalyl Diisothiocyanate
}

\author{
Jonathan Pfeiffer, ${ }^{[\mathrm{a}]}$ Clemens Trost, ${ }^{[\mathrm{a}]}$ Anna Pachkovska, ${ }^{[\mathrm{a}]}$ and Frank Tambornino*[a] \\ [a] Fachbereich Chemie, Wissenschaftlichen Zentrum für Materialwissenschaften (WZMW) \\ Philipps-Universität Marburg \\ Hans-Meerwein-Straße 4, D-35043 Marburg, Germany \\ E-mail: frank.tambornino@chemie.uni-marburg.de
}

\section{Contents}

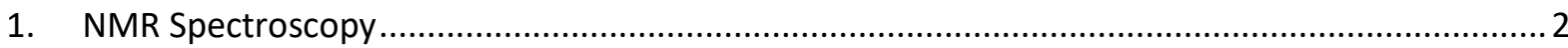

2. IR Spectroscopy

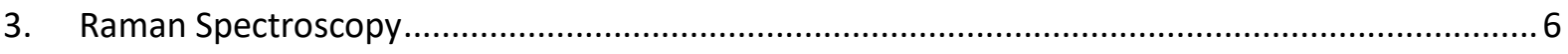

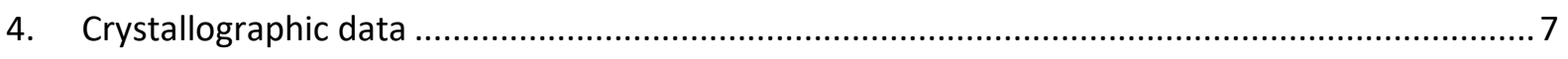

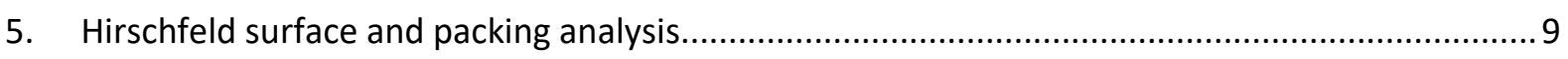

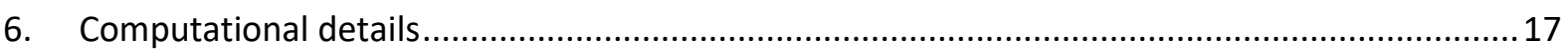




\section{NMR Spectroscopy}

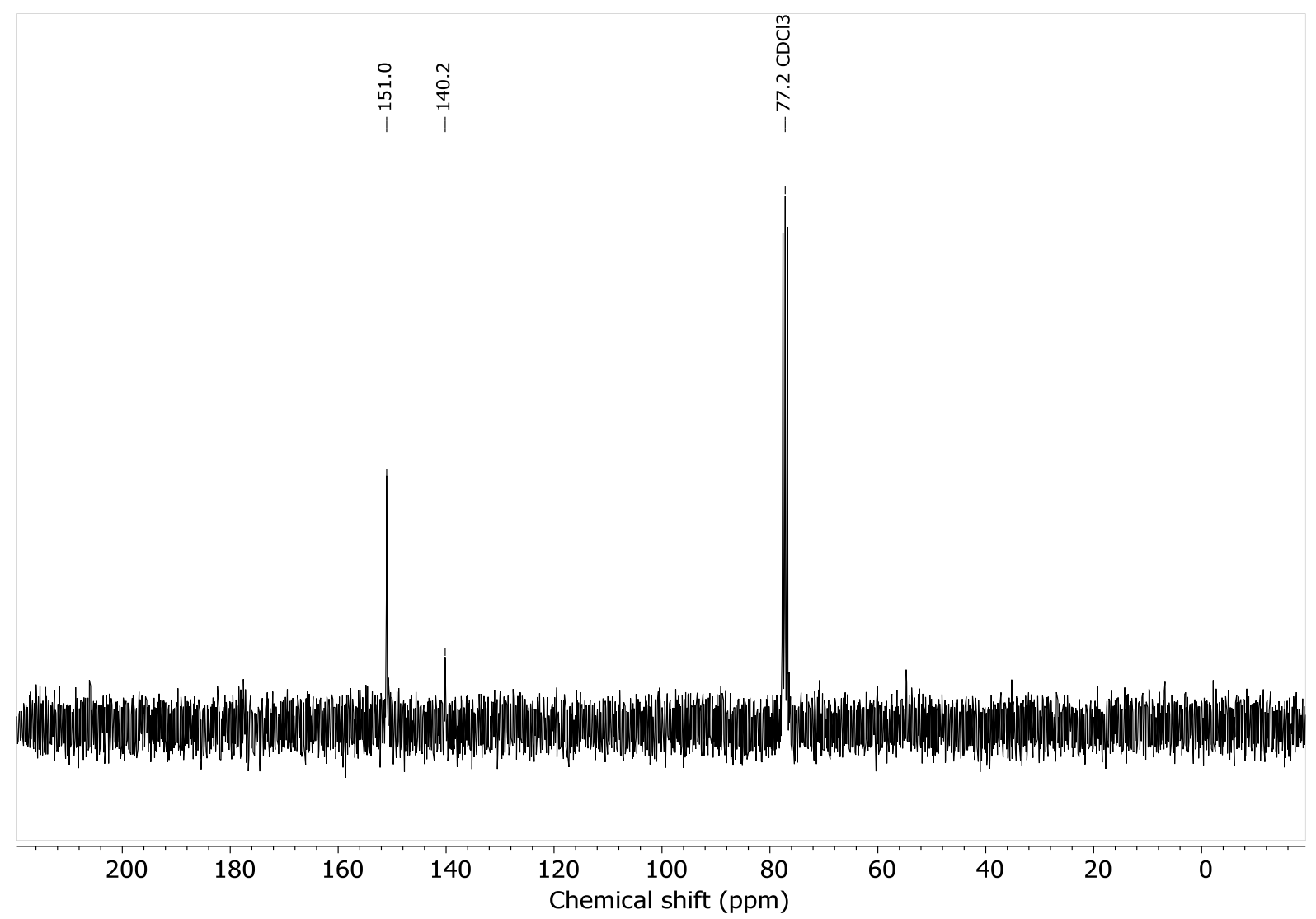

Figure $\mathrm{S} 1:{ }^{13} \mathrm{C}$ NMR spectrum of carbonyl diisothiocyanate in $\mathrm{CDCl}_{3}$. 


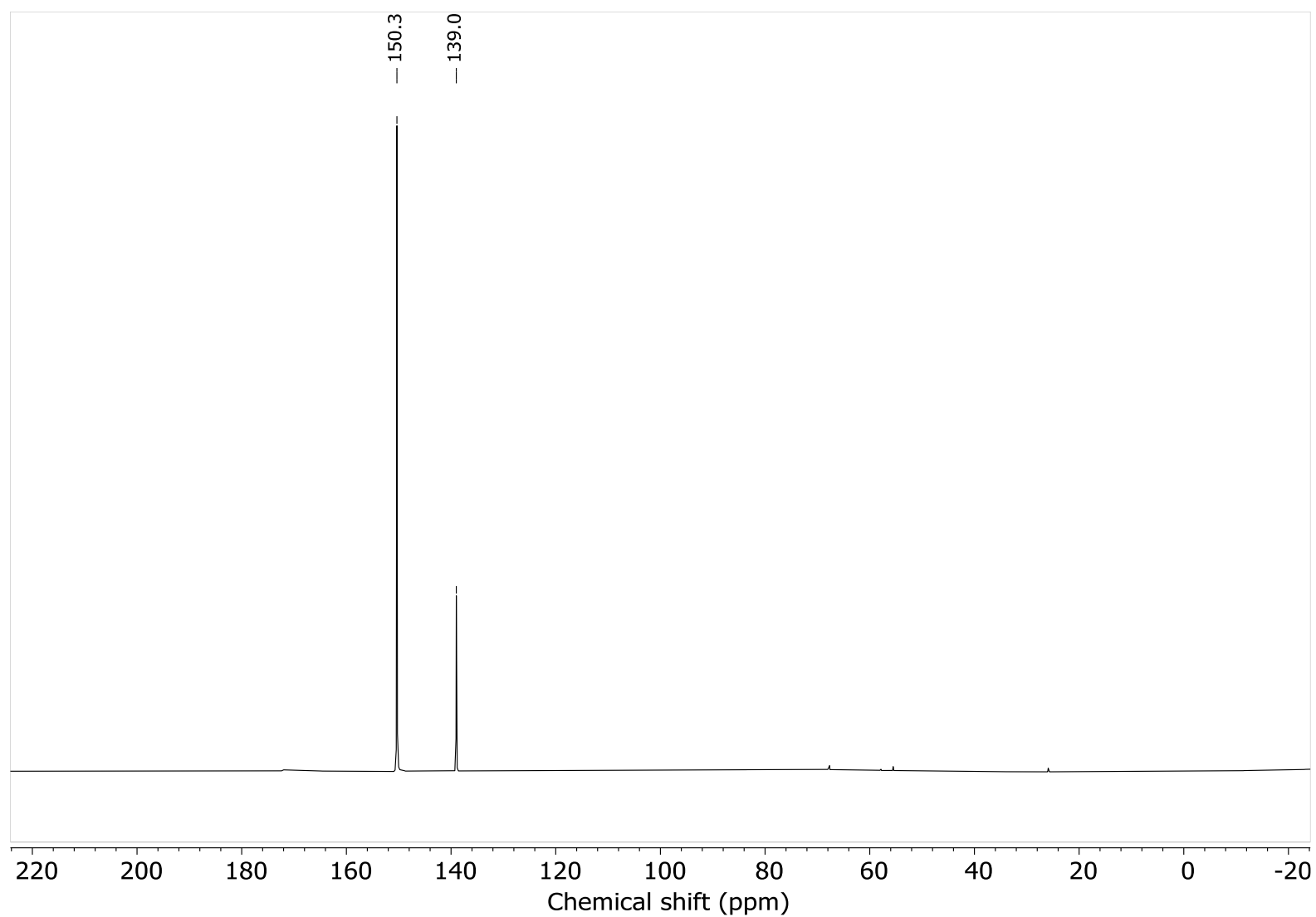

Figure S2: ${ }^{13} \mathrm{C}$ NMR spectrum of pure carbonyl diisothiocyanate without solvent.

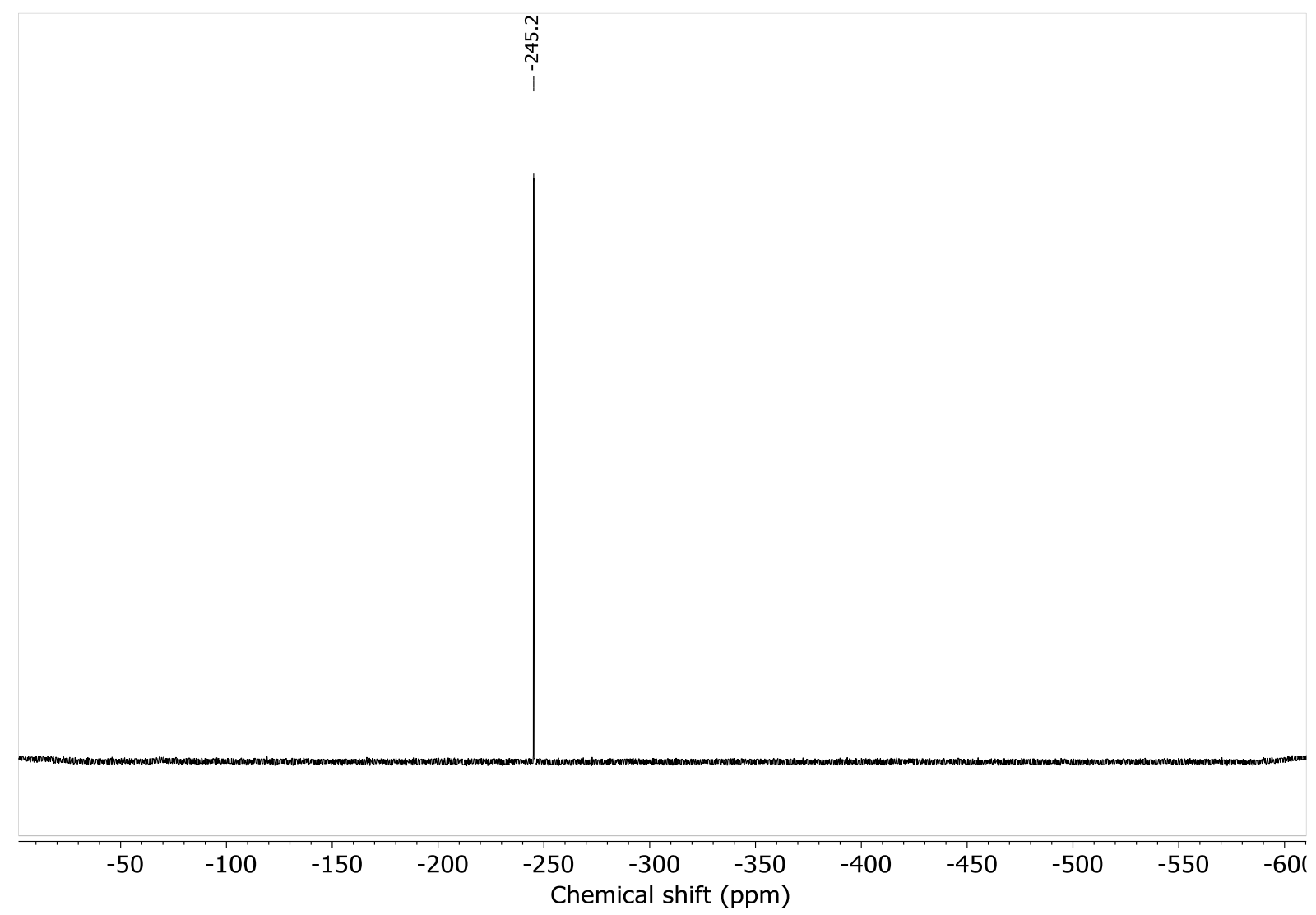

Figure S3: ${ }^{15} \mathrm{~N}$ NMR spectrum of pure carbonyl diisothiocyanate without solvent.

S.I. 3 


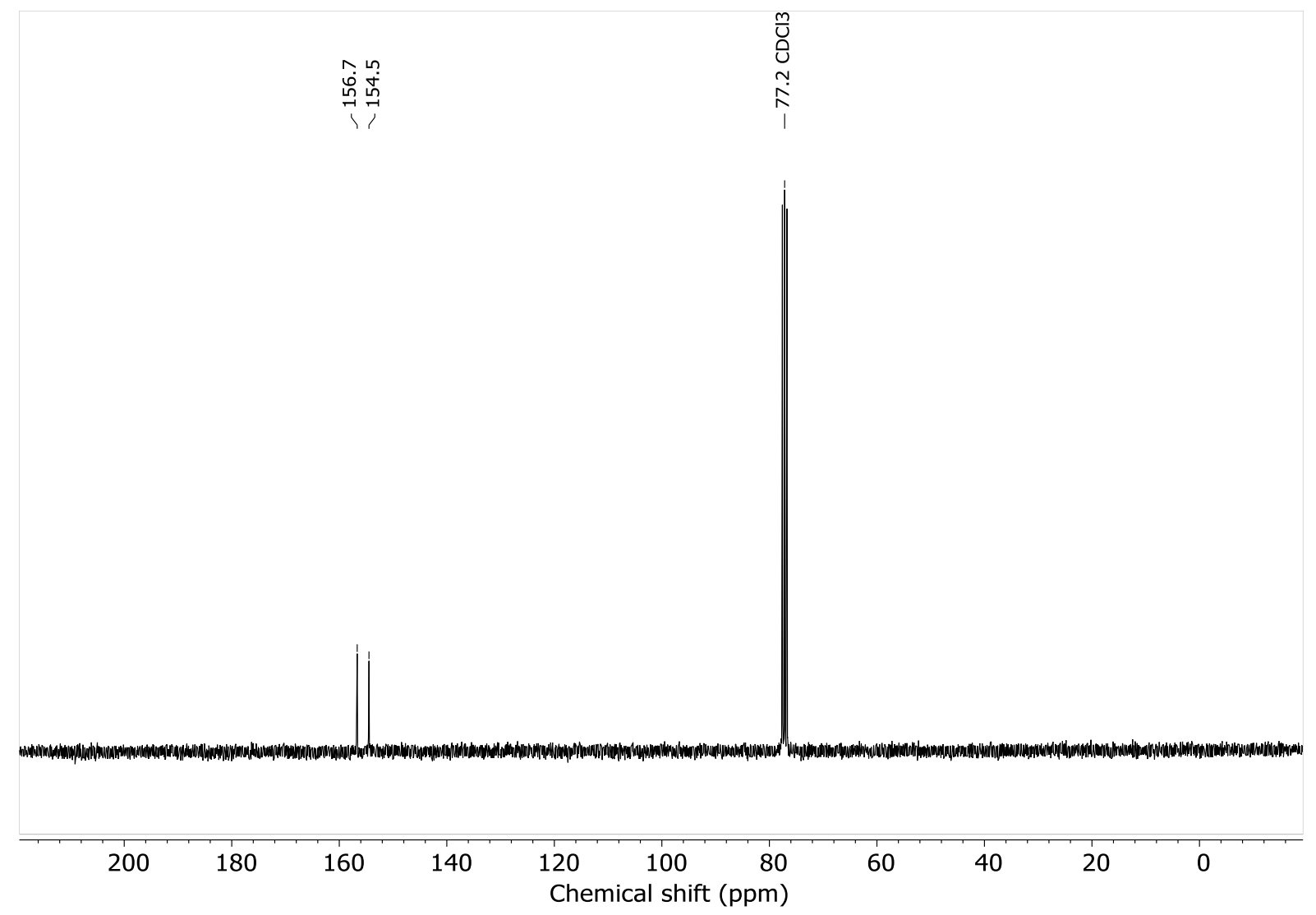

Figure $\mathrm{S} 4:{ }^{13} \mathrm{C}$ NMR spectrum of oxalyl diisothiocyanate in $\mathrm{CDCl}_{3}$.

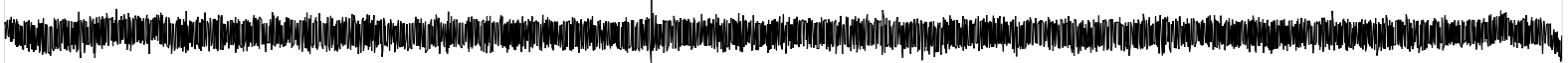

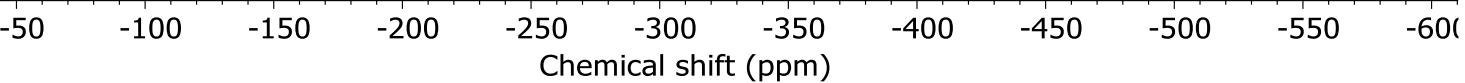

Figure S5: ${ }^{15} \mathrm{~N}$ NMR spectrum of oxalyl diisothiocyanate in THF-d8.

S.I. 4 


\section{IR Spectroscopy}

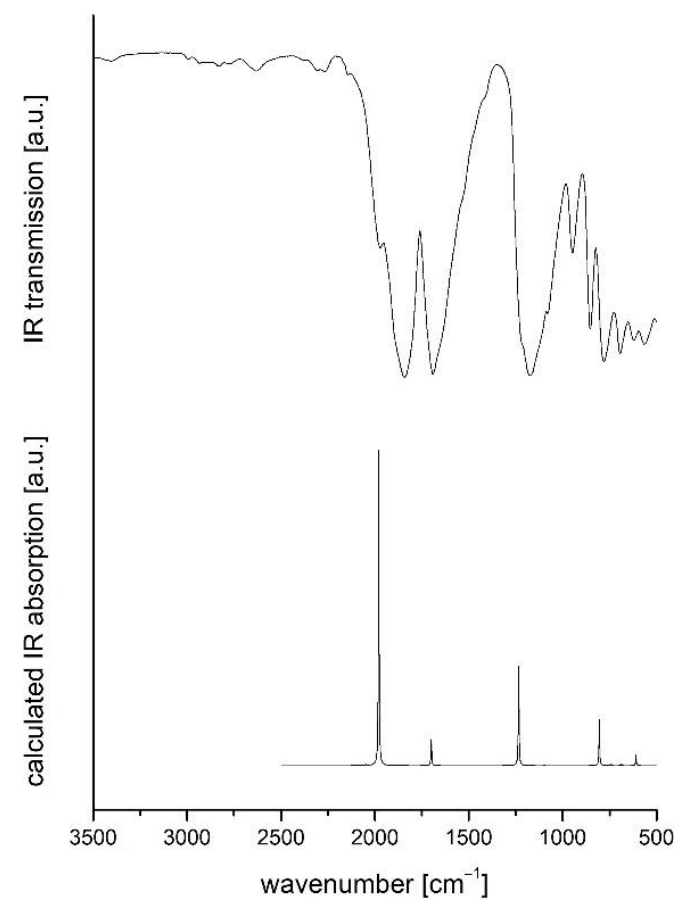

Figure S6: Top: Measured room-temperature ATR-IR spectrum of carbonyl diisothiocyanate. Bottom: Calculated solid-state IR spectrum of carbonyl diisothiocyanate.

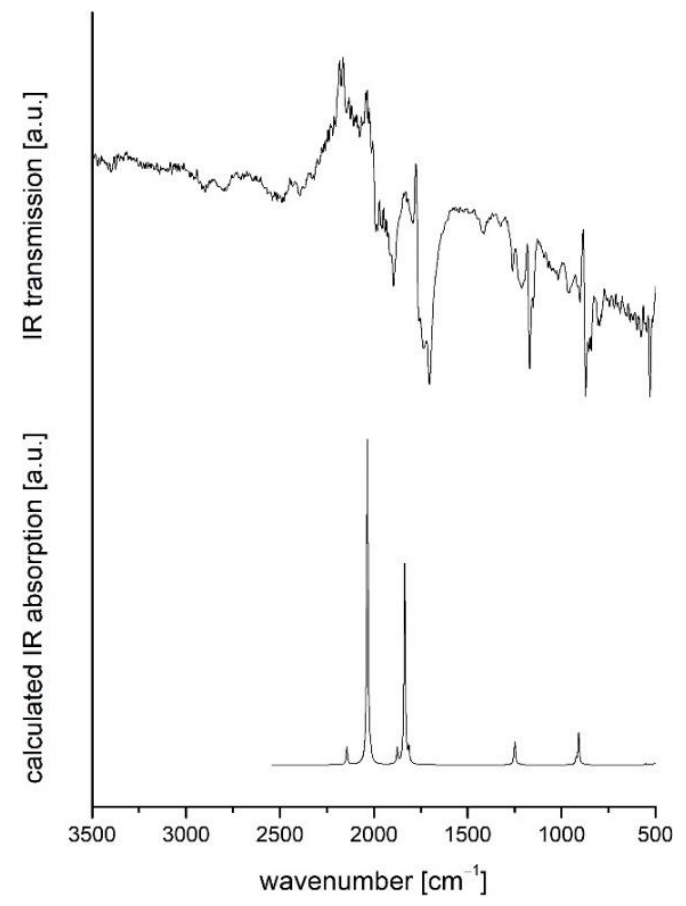

Figure S7: Top: Measured room-temperature ATR-IR spectrum of oxalyl diisothiocyanate. Bottom: Calculated solid-state IR spectrum of oxalyl diisothiocyanate. 


\section{Raman Spectroscopy}

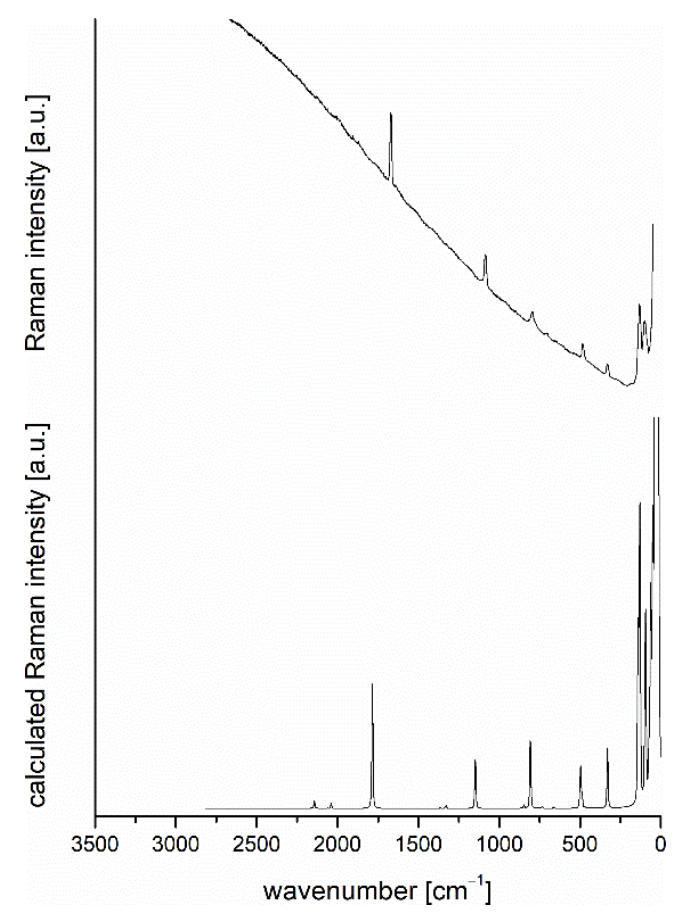

Figure S8: Measured Raman spectrum of carbonyl diisothiocyanate at $-80{ }^{\circ} \mathrm{C}$. Bottom: Calculated solid-state Raman spectrum of carbonyl diisothiocyanate.

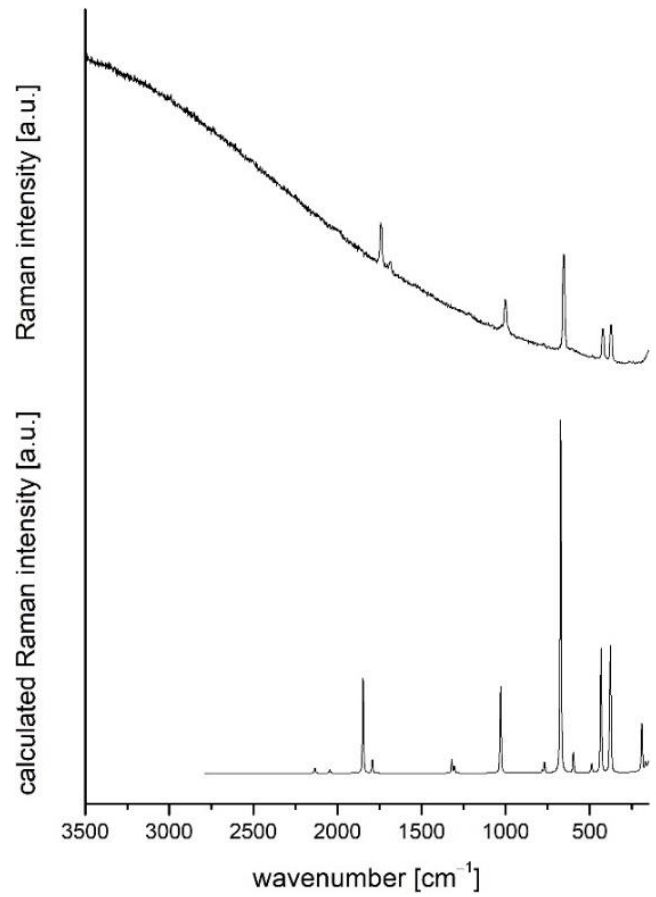

Figure S9: Top: Measured room-temperature Raman spectrum of oxalyl diisothiocyanate. Bottom: Calculated solid-state Raman spectrum of oxalyl diisothiocyanate. 


\section{Crystallographic data}

Table S1: Selected X-ray data collection and refinement parameters for carbonyl diisothiocyanate and oxalyl diisothiocyanate.

\begin{tabular}{|c|c|c|}
\hline & Carbonyl diisothiocyanate & Oxalyl diisothiocyanate \\
\hline Formula & $\mathrm{C}_{3} \mathrm{~N}_{2} \mathrm{O}_{1} \mathrm{~S}_{2}$ & $\mathrm{C}_{4} \mathrm{~N}_{2} \mathrm{O}_{2} \mathrm{~S}_{2}$ \\
\hline $\mathrm{CCDC}$ & 2080495 & 2080496 \\
\hline $\mathrm{FW} / \mathrm{g} \mathrm{mol}^{-1}$ & 144.17 & 688.72 \\
\hline Crystal system & monoclinic & orthorhombic \\
\hline Space group & $P 2_{1} / c($ no. 14$)$ & $P b c a($ no. 61) \\
\hline$a / \AA$ & $20.5118(13)$ & $5.4245(2)$ \\
\hline$b / \AA$ & $3.84410(10)$ & $20.2875(12)$ \\
\hline$c / \AA$ & $15.9661(11)$ & $6.1642(4)$ \\
\hline$\alpha / \circ$ & 90 & 90 \\
\hline$\beta / \circ$ & $112.464(5)$ & 90 \\
\hline$\gamma /{ }^{\circ}$ & 90 & 90 \\
\hline$V / \AA^{3}$ & $1163.39(12)$ & $678.37(6)$ \\
\hline$Z$ & 8 & 4 \\
\hline Radiation, $\lambda / \AA$ & 1.54186 & 1.54186 \\
\hline Temp / K & 200 & 100 \\
\hline$\rho_{\text {calc }} / \mathrm{g} \mathrm{cm}^{-3}$ & 1.646 & 1.686 \\
\hline$\mu / \mathrm{mm}^{-1}$ & 7.468 & 6.636 \\
\hline$\theta$ range $/ \circ$ & $2.995-65.479$ & $4.359-69.919$ \\
\hline Index range & $-24 \leq h \leq 23,-4 \leq k \leq 3,-15 \leq l \leq 18$ & $-4 \leq h \leq 6,-24 \leq k \leq 24,-7 \leq l \leq 7$ \\
\hline Reflections collected & 17346 & 15245 \\
\hline Independent reflections & $2010 / 1454$ & $643 / 504$ \\
\hline Parameters & 147 & 46 \\
\hline $\mathrm{R}_{\text {int }} / \mathrm{R}_{(\sigma)}$ & $5.47 / 3.28$ & $6.16 / 2.45$ \\
\hline$R 1 / w R 2,{ }^{[\mathrm{a}]} I \geq 2 \sigma I(\%)$ & $3.75 / 9.58$ & $3.22 / 7.21$ \\
\hline$R 1 / w R 2,{ }^{[\mathrm{a}]}$ all data $(\%)$ & $5.34 / 10.47$ & $4.57 / 7.44$ \\
\hline GOF & 0.903 & 0.961 \\
\hline Twin law & $\begin{array}{lllllllll}-1 & 0 & -1 & 0 & -1 & 0 & 0 & 0 & 1\end{array}$ & - \\
\hline BASF & $0.251(3)$ & - \\
\hline
\end{tabular}


Table S2: Selected powder X-ray data collection and Rietveld refinement parameters for oxalyl diisothiocyanate.

\begin{tabular}{|c|c|}
\hline & Oxalyl diisothiocyanate \\
\hline Formula & $\mathrm{C}_{4} \mathrm{~N}_{2} \mathrm{O}_{2} \mathrm{~S}_{2}$ \\
\hline CCDC & 2080497 \\
\hline $\mathrm{Fw} / \mathrm{g} \mathrm{mol}^{-1}$ & 688.72 \\
\hline Crystal system & orthorhombic \\
\hline Space group & Pbca (no. 61) \\
\hline$a / \AA$ & $5.4757(2)$ \\
\hline$b / \AA$ & $20.523(7)$ \\
\hline$c / \AA$ & $6.3625(2)$ \\
\hline$\alpha /{ }^{\circ}$ & 90 \\
\hline$\beta / \circ$ & 90 \\
\hline$\gamma / \circ$ & 90 \\
\hline$V / \AA^{3}$ & $715.004(4)$ \\
\hline$Z$ & 4 \\
\hline Radiation, $\lambda / \AA$ & 1.54186 \\
\hline Temp / K & 298 \\
\hline$R_{\mathrm{wp}} / \%$ & 5.902 \\
\hline$R_{\mathrm{p}} / \%$ & 4.469 \\
\hline$R_{\text {Bragg }} / \%$ & 3.061 \\
\hline GoF & 2.284 \\
\hline Starting angle measured $/{ }^{\circ} 2 \theta$ & 5 \\
\hline Final angle measured $/{ }^{\circ} 2 \theta$ & 90 \\
\hline Starting angle refined $/{ }^{\circ} 2 \theta$ & 20 \\
\hline Final angle refined $/{ }^{\circ} 2 \theta$ & 70 \\
\hline Step width $/{ }^{\circ} 2 \theta$ & 0.015 \\
\hline Refined Parameters ${ }^{[\mathrm{a}]}$ & 25 \\
\hline Background Parameters & 6 \\
\hline Profile Parameters & 9 \\
\hline
\end{tabular}




\section{Hirschfeld surface and packing analysis}

\section{Carbonyl diisothiocyanate (1)}

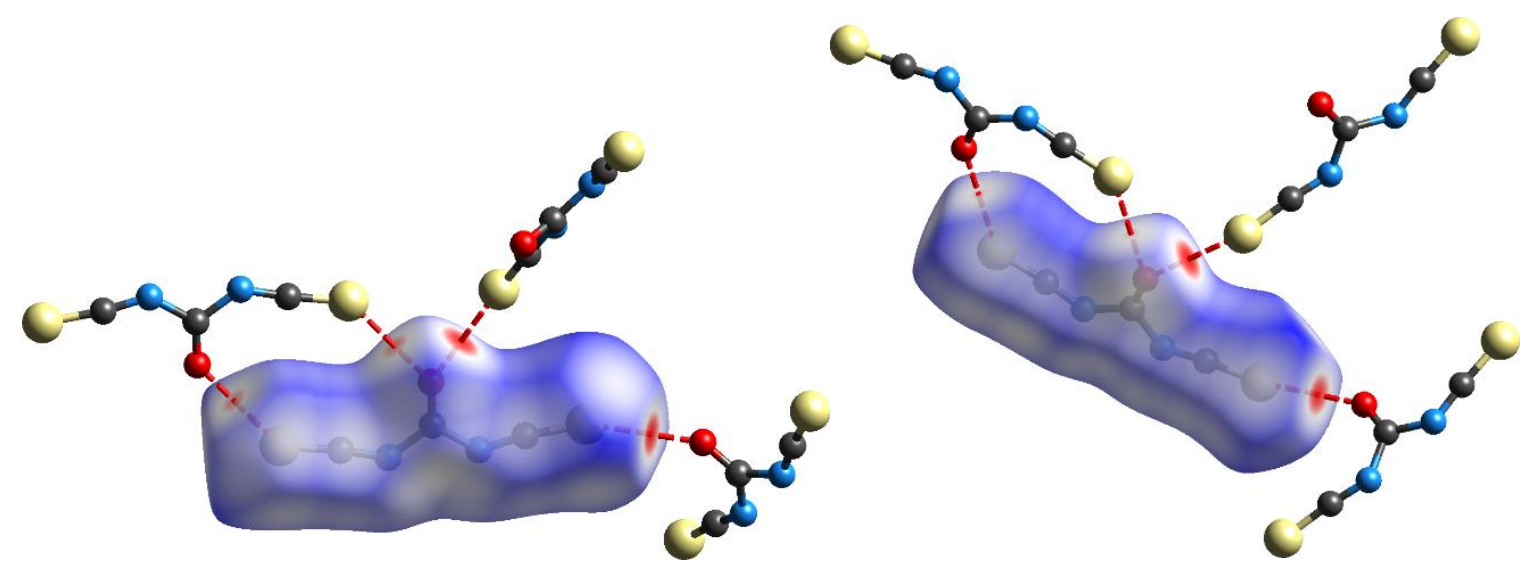

Figure S10: Hirschfeld surfaces for carbonyl diisothiocyanate crystallographic individuum 1 (left) and individuum 2 (right). Red colour on the surface indicates an intermolecular contact shorter than the sum of van-der-Waals radii and short contacts are highlighted by red dashed lines.

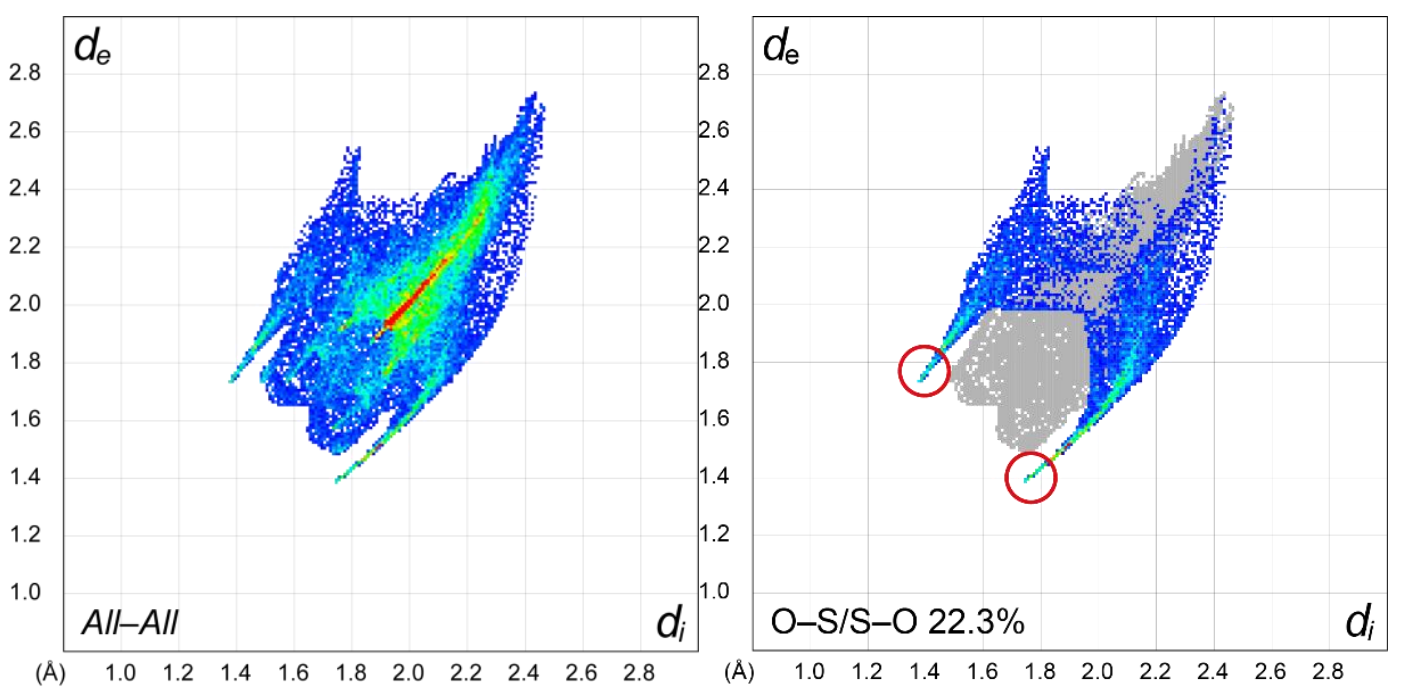

Figure S11: 2D fingerprint plots for all (left) and $\mathrm{O}-\mathrm{S} / \mathrm{S}-\mathrm{O}$ (right) contacts. The two spikes (red circles) are the short $\mathrm{O}-\mathrm{S}$ contacts. 


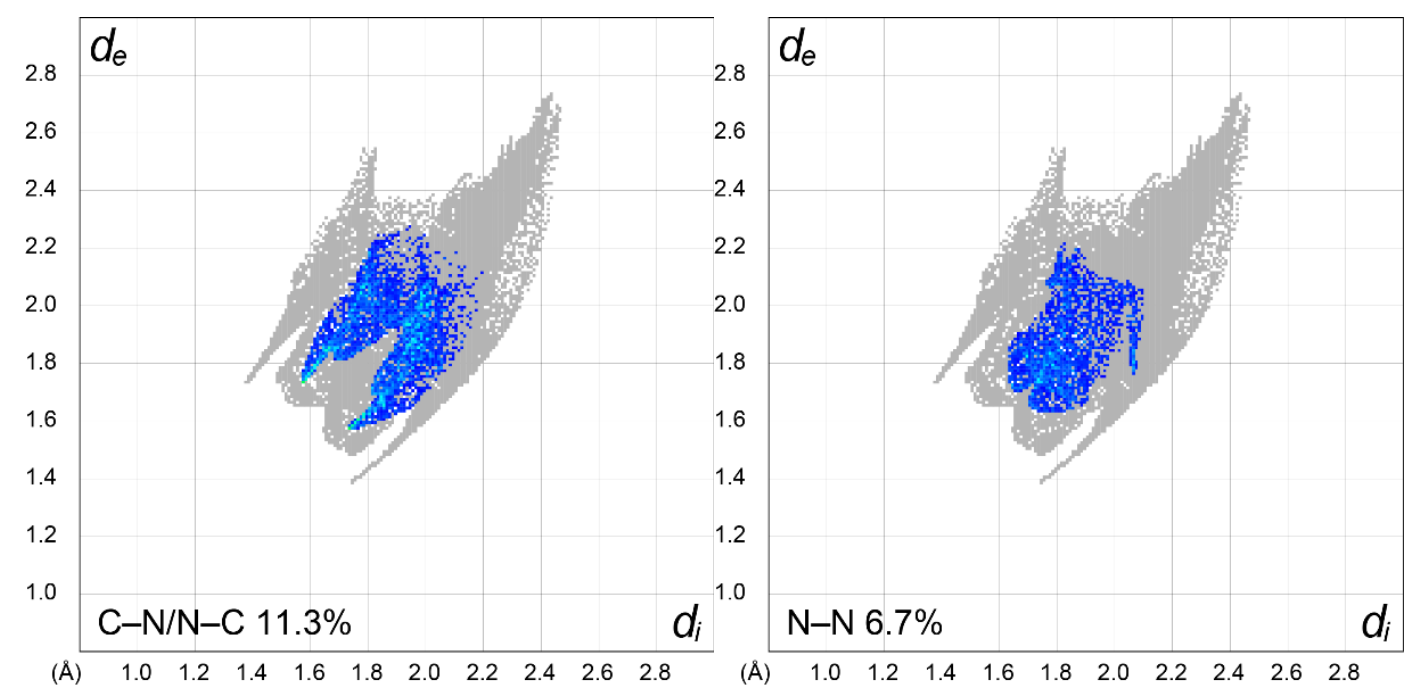

Figure S12: 2D fingerprint plots for $\mathrm{C}-\mathrm{N} / \mathrm{N}-\mathrm{C}$ (left) and $\mathrm{N}-\mathrm{N}$ (right) contacts.
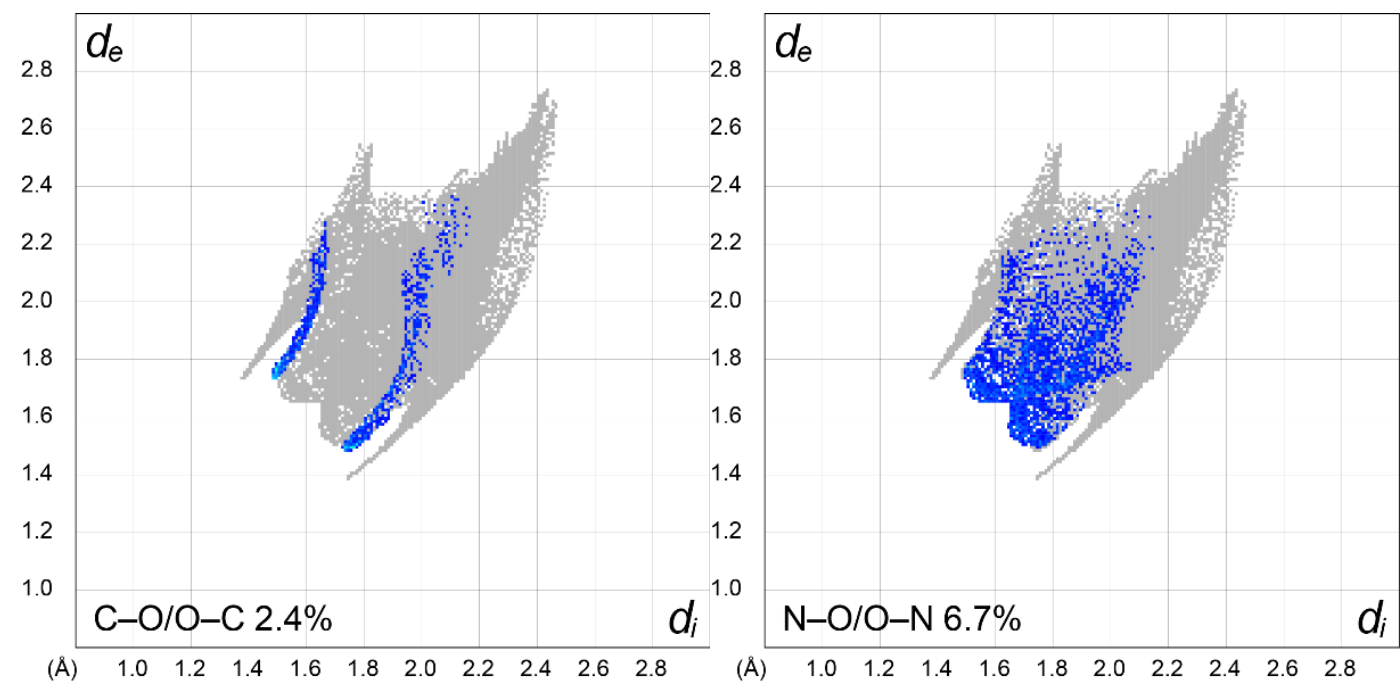

Figure S13: 2D fingerprint plots for $\mathrm{C}-\mathrm{O} / \mathrm{O}-\mathrm{C}$ (left) and $\mathrm{N}-\mathrm{O} / \mathrm{O}-\mathrm{N}$ (right) contacts. 


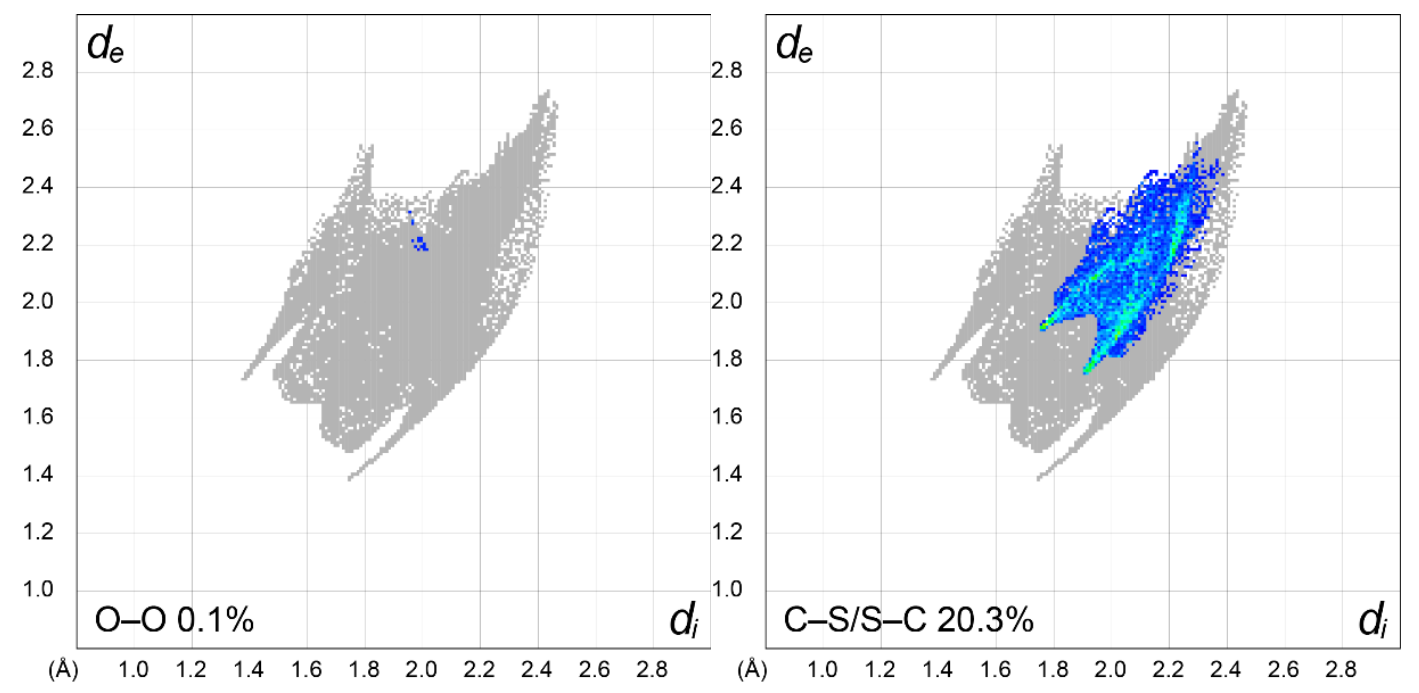

Figure S14: 2D fingerprint plots for $\mathrm{O}-\mathrm{O}$ (left) and $\mathrm{C}-\mathrm{S} / \mathrm{S}-\mathrm{C}$ (right) contacts.
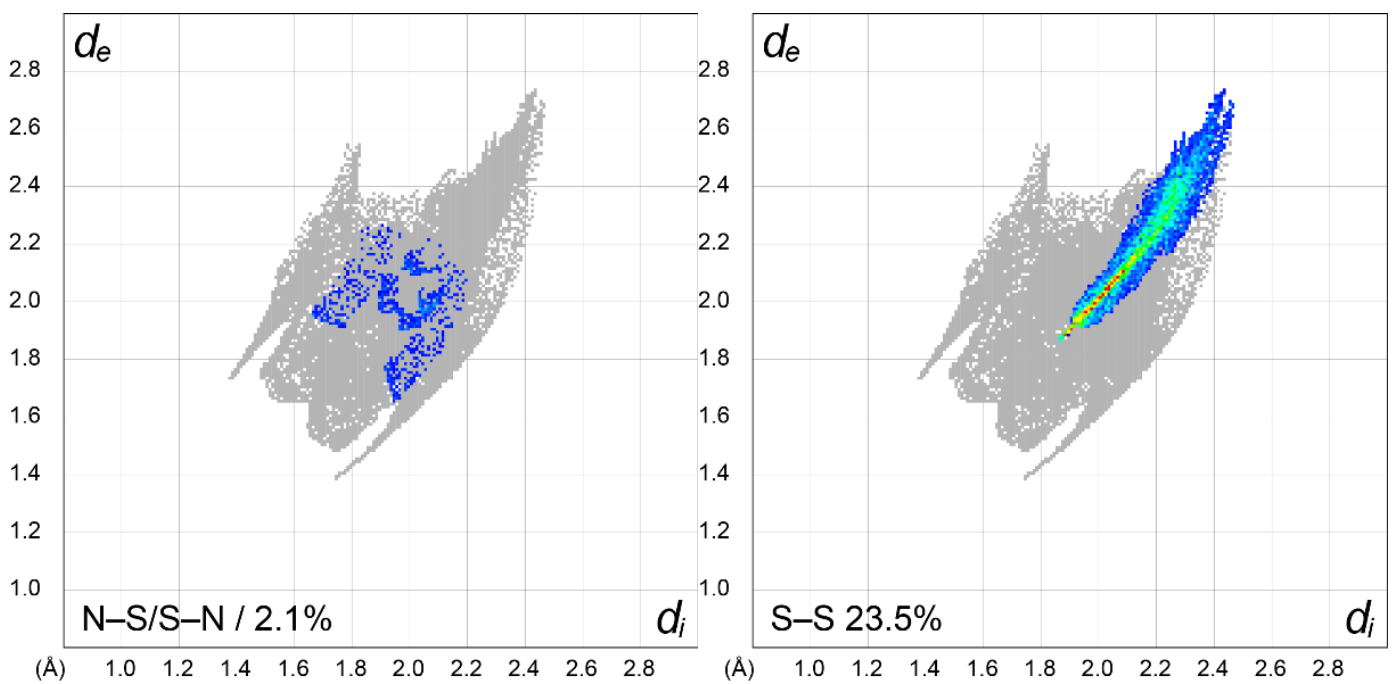

Figure S15: 2D fingerprint plots for N-S/S-N (left) and S-S (right) contacts. The latter are the main contact area on the Hirschfeld surface and lie in the range of the sum of their van-der-Waals radii. 


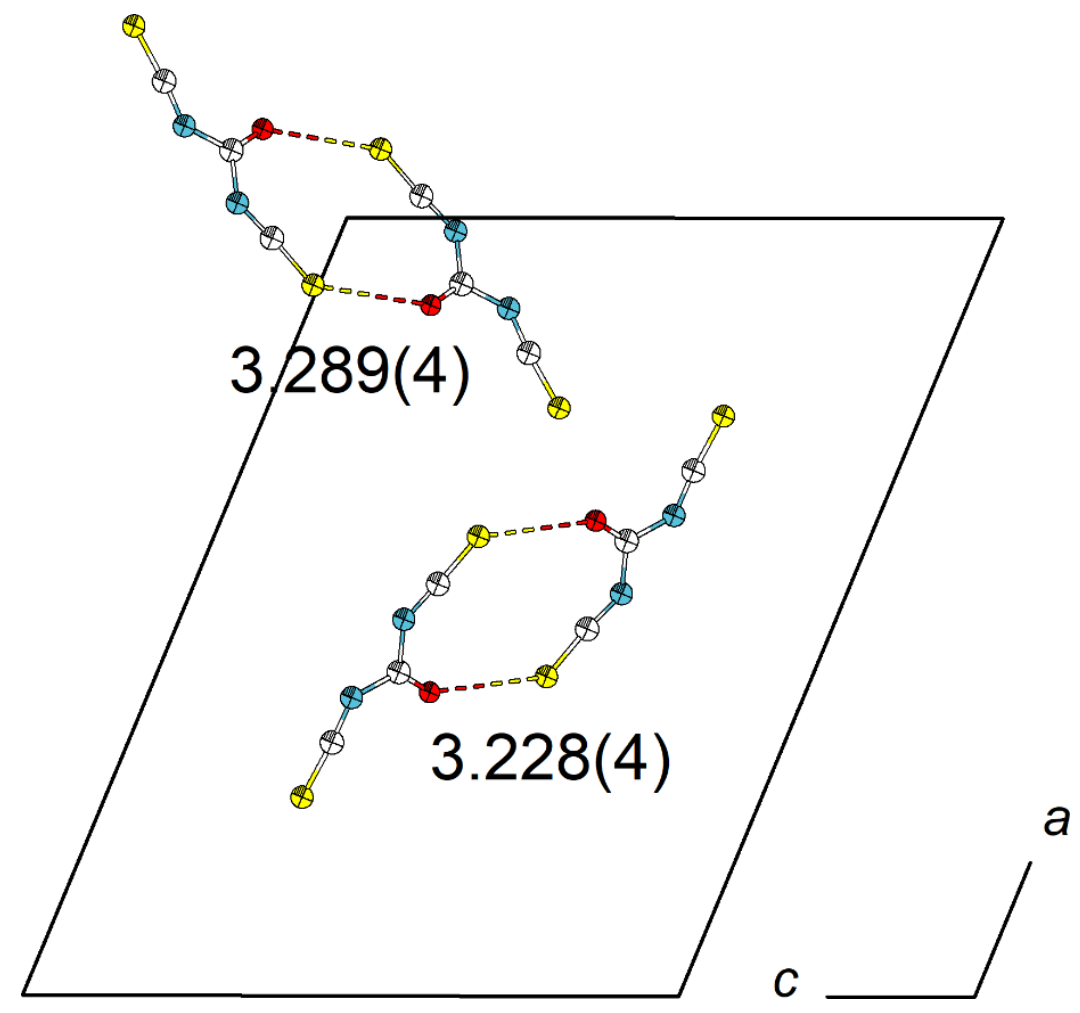

Figure S16: Bonding between the crystallographically independent carbonyl diisothiocyanate molecules to their respective symmetry generated pair. Short contacts are drawn as dashed lines.

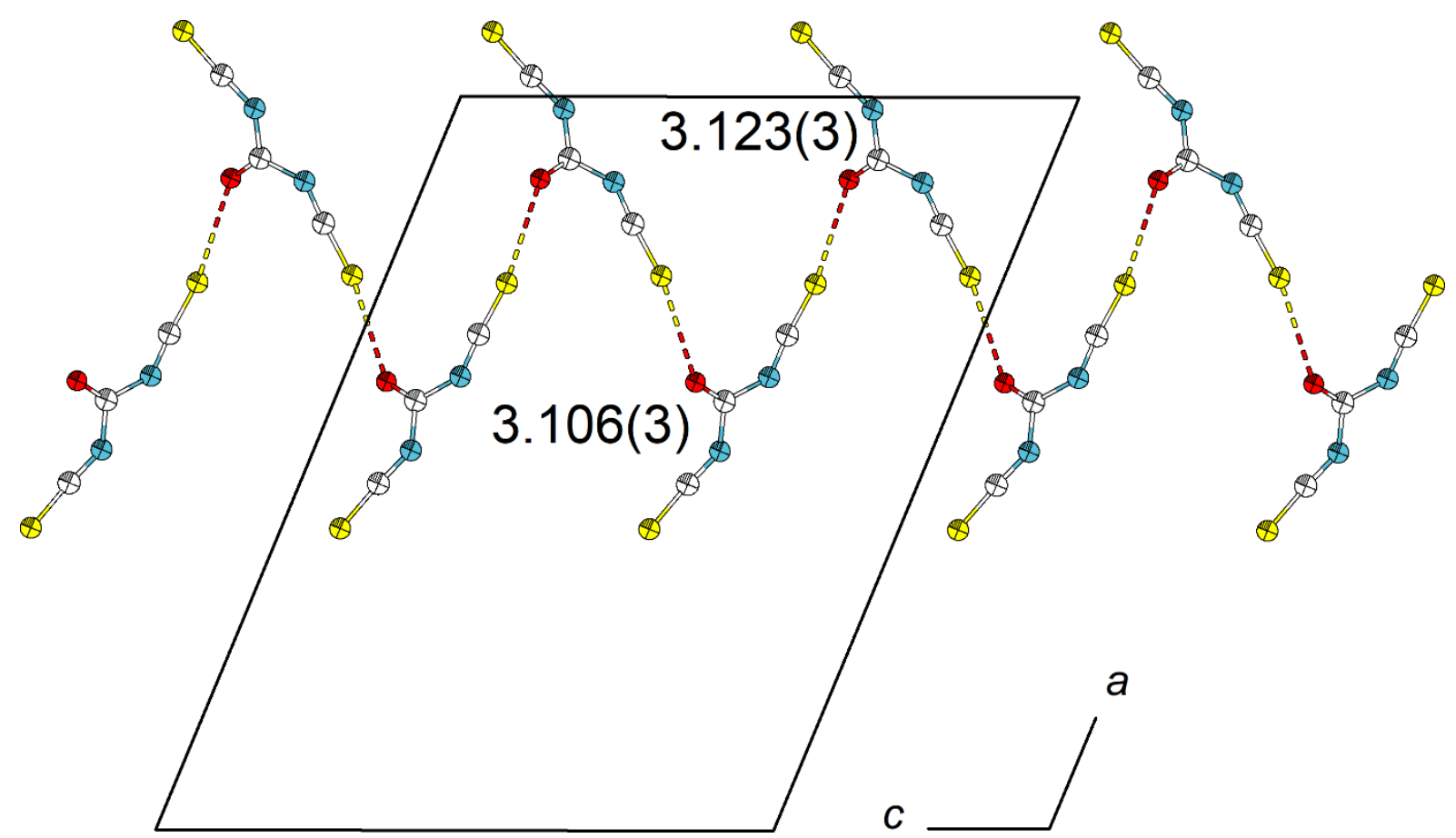

Figure S17: Bonding between the crystallographically independent molecules of carbonyl diisothiocyanate forming a puckered zig-zag-chain along [001]. 


\section{Oxalyl diisothiocyanate (2)}

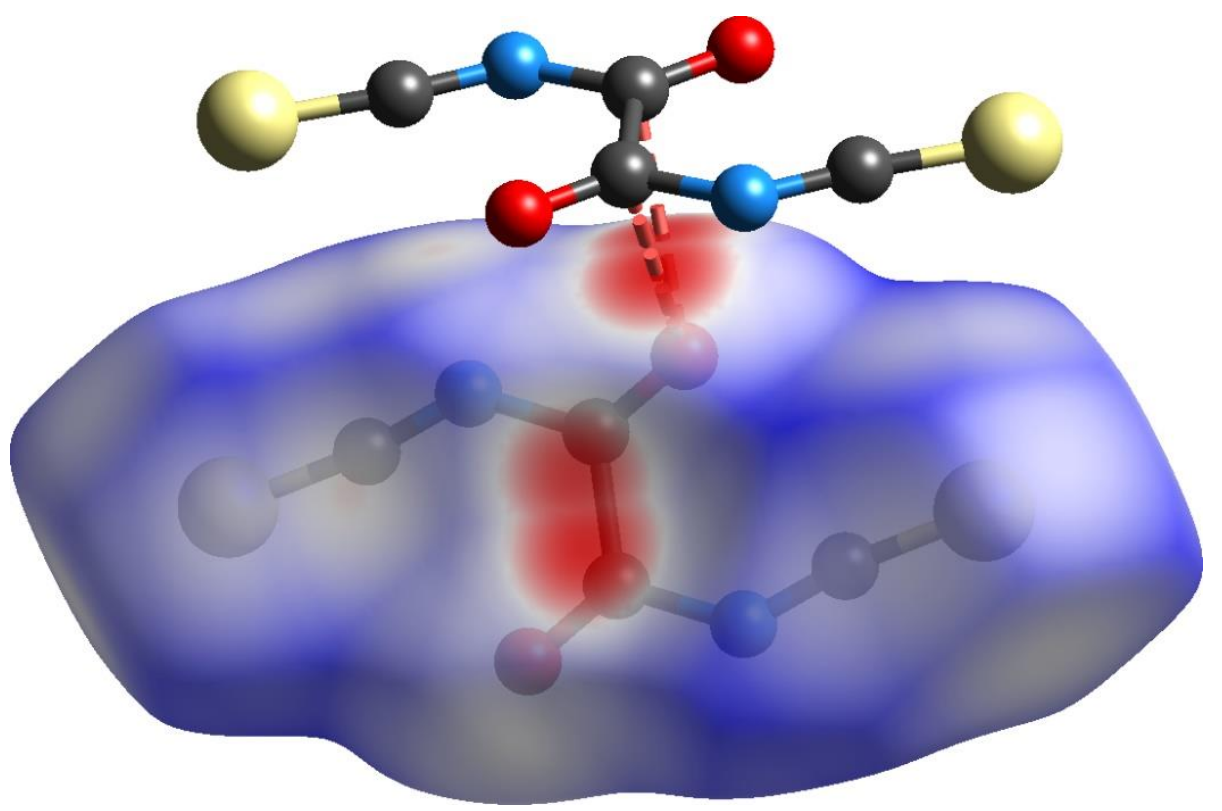

Figure S18: Hirschfeld surface for oxalyl diisothiocyanate. Red indicates an intermolecular contact shorter than the sum of van-der-Waals radii and short contacts are highlighted as red dashed lines.
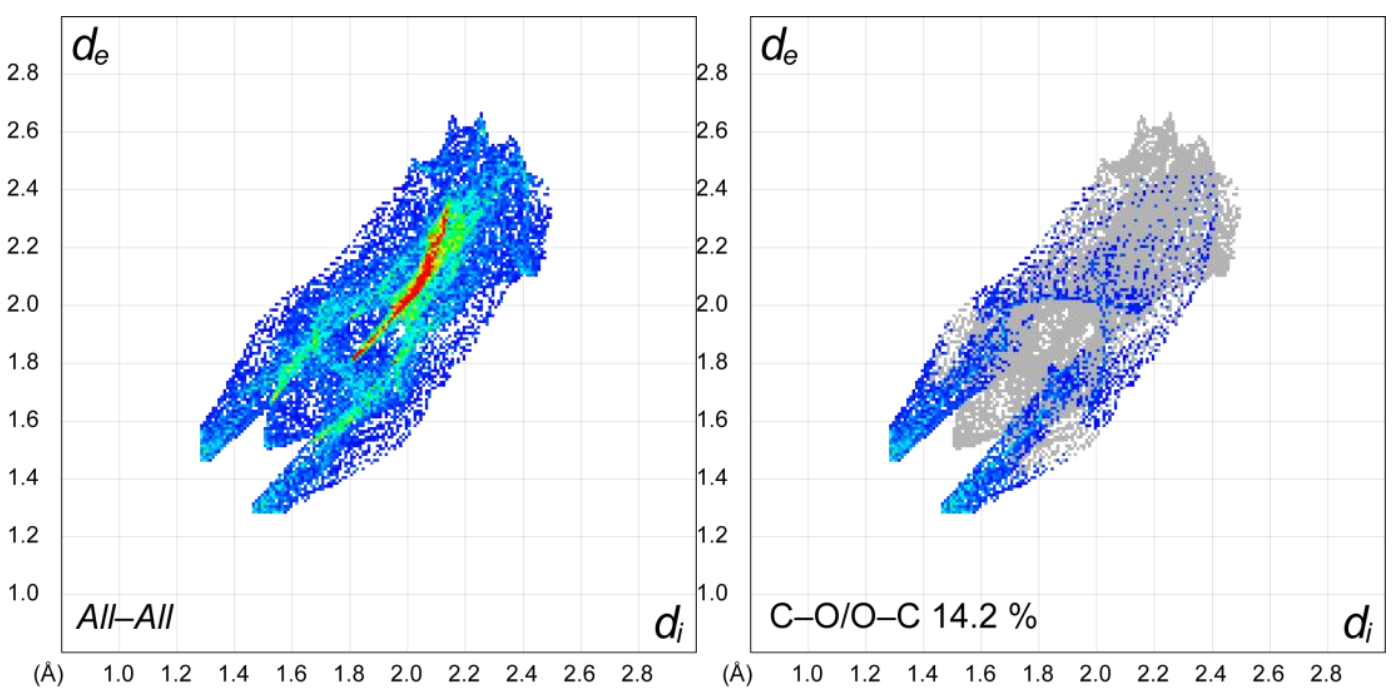

Figure S19: 2D fingerprint plots for all (left) and $\mathrm{C}-\mathrm{O} / \mathrm{O}-\mathrm{C}$ (right) contacts in oxalyl diisothiocyanate. The two spikes (red circles) are the short $\mathrm{C}-\mathrm{O}$ contacts which are closer than the sum of the van-derWaals radii. 

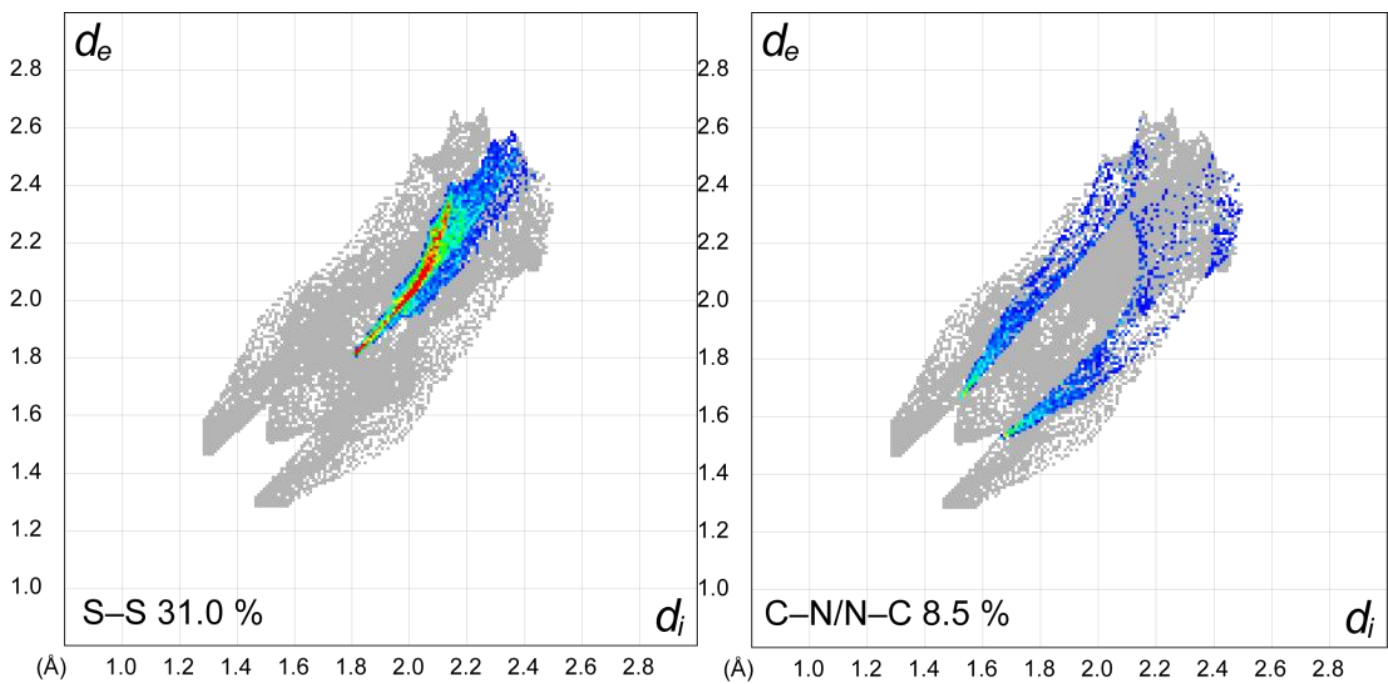

Figure S20: 2D fingerprint plots for $\mathrm{S}-\mathrm{S}$ (left) and $\mathrm{C}-\mathrm{N} / \mathrm{N}-\mathrm{C}$ (right) contacts in oxalyl diisothiocyanate. $\mathrm{S}-\mathrm{S}$ contacts are responsible for interplane packing.
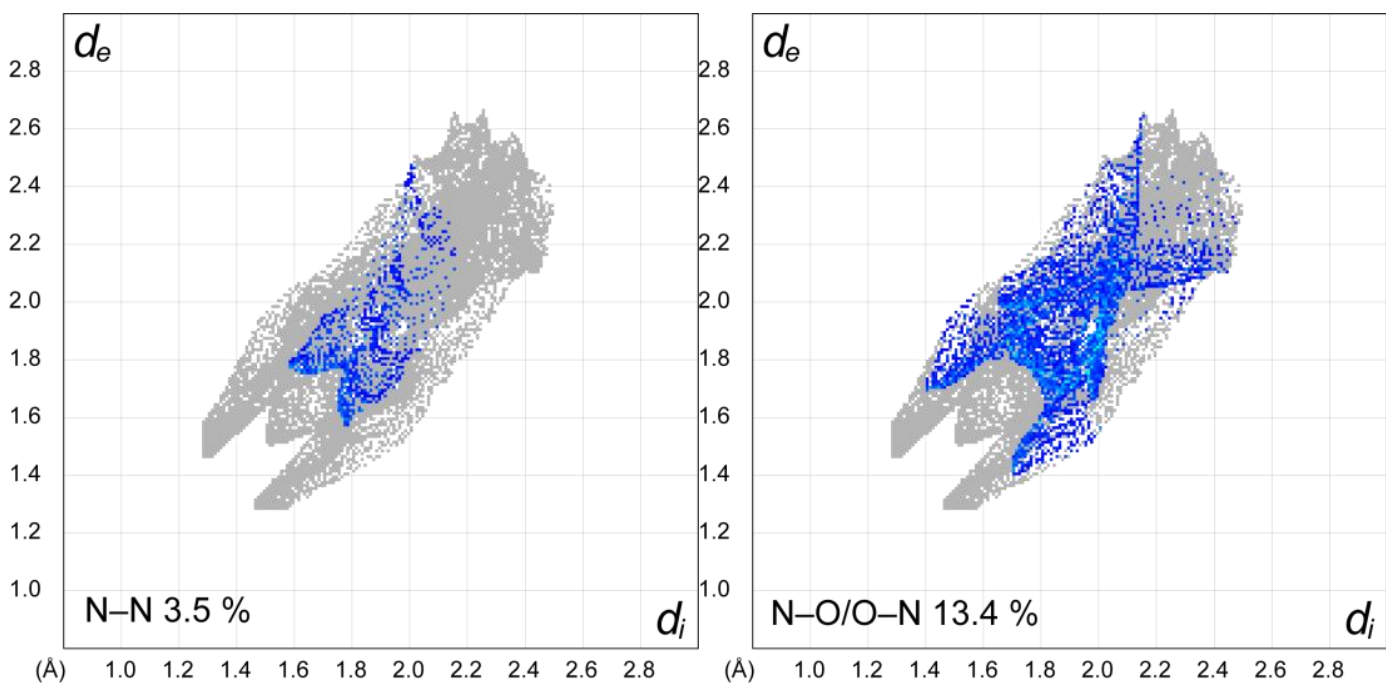

Figure S21: 2D fingerprint plots for $\mathrm{N}-\mathrm{N}$ (left) and $\mathrm{N}-\mathrm{O} / \mathrm{O}-\mathrm{N}$ (right) contacts in oxalyl diisothiocyanate. 


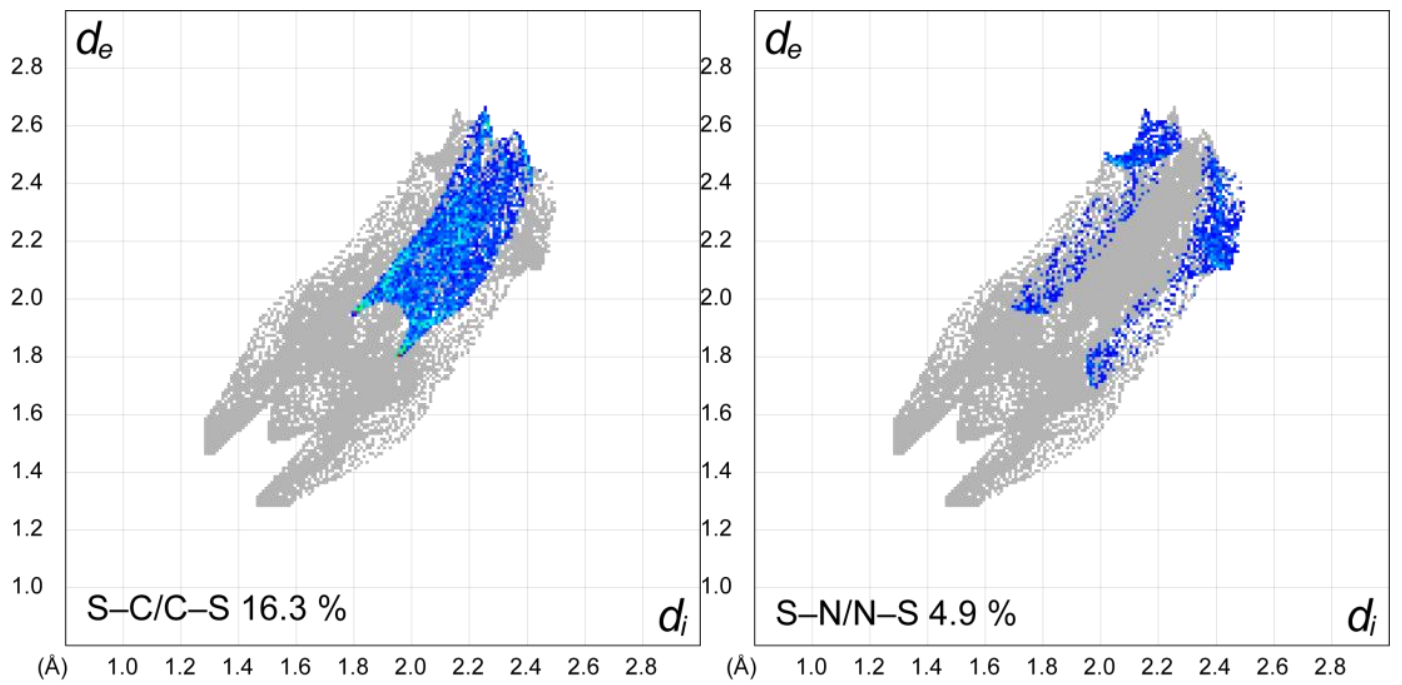

Figure S22: 2D fingerprint plots for $\mathrm{S}-\mathrm{C} / \mathrm{C}-\mathrm{S}$ (left) and $\mathrm{S}-\mathrm{N} / \mathrm{N}-\mathrm{S}$ (right) contacts in oxalyl diisothiocyanate.

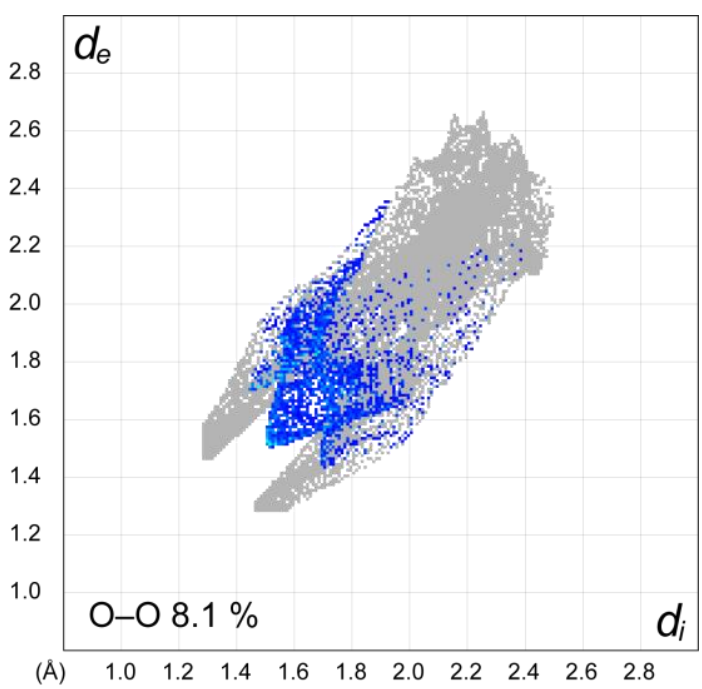

Figure S23: 2D fingerprint plots for $\mathrm{O}-\mathrm{O}$ contacts in oxalyl diisothiocyanate. 


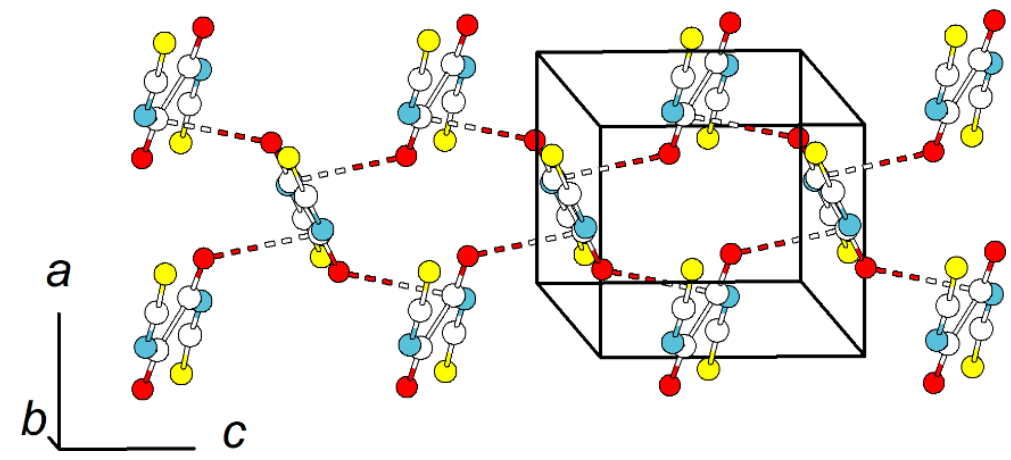

Figure S24: Section of the crystal structure of oxalyl diisothiocyanate viewed approximately along [010] emphasizing the interconnection of individual molecules. $\mathrm{C}-\mathrm{O}$ short contacts are shown as dashed lines. The $\mathrm{C}-\mathrm{O}$ distance is $2.769(2) \AA$.

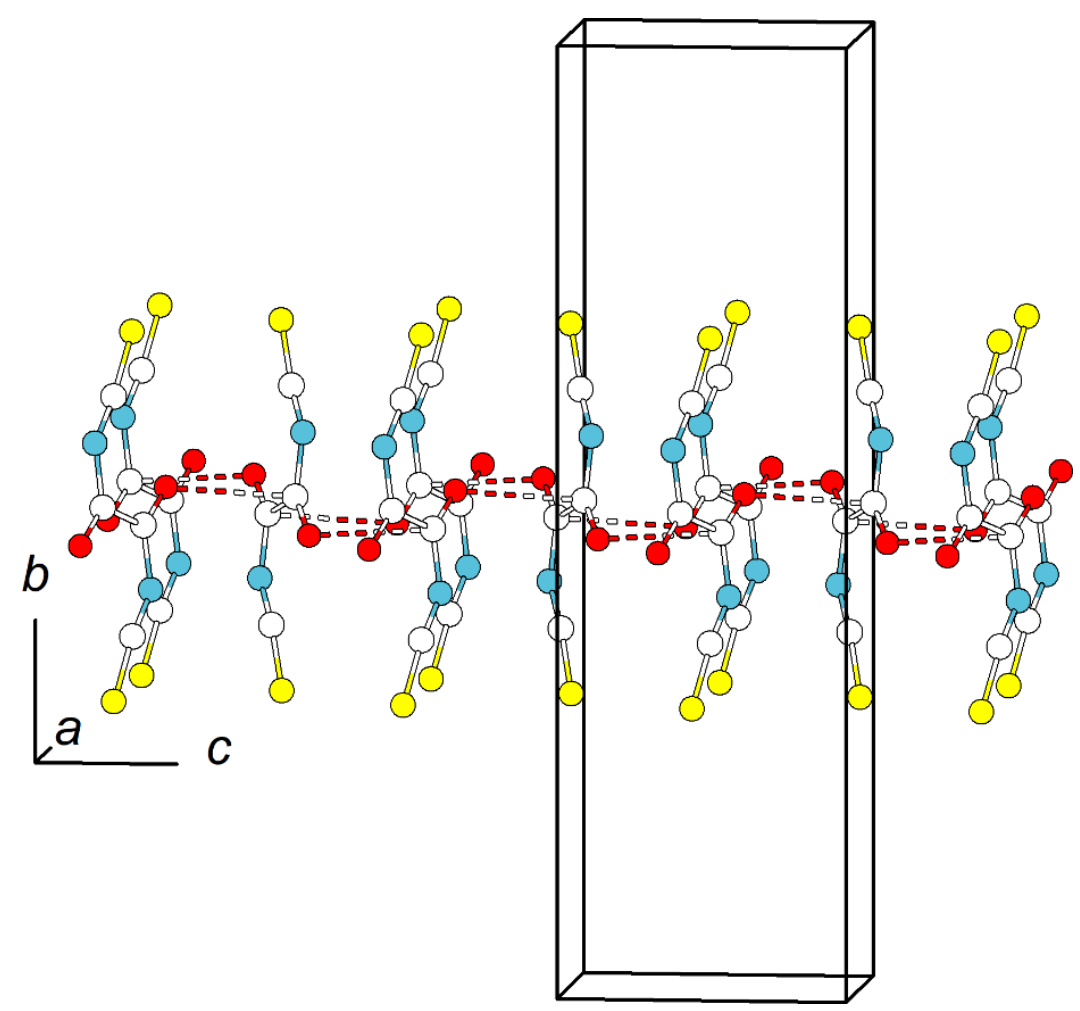

Figure S25: Section of the crystal structure of oxalyl diisothiocyanate viewed approximately along [100] emphasizing the interconnection of individual molecules and layer formation. $\mathrm{C}-\mathrm{O}$ short contacts are shown as dashed lines. 


\section{Computational details}

Table S3: Lattice parameters and atomic coordinates of the optimized solid-state structure of carbonyl diisothiocyanate.

\begin{tabular}{|c|c|c|c|c|}
\hline $\begin{array}{l}\text { Lattice } \\
\text { parameter }\end{array}$ & $a$ & $b$ & $c$ & $\beta$ \\
\hline & 21.33227251 & 4.09862195 & 16.62696050 & 112.951696 \\
\hline Atom & $x$ & $y$ & & $z$ \\
\hline S1 & $8.198325514532 \mathrm{E}-02$ & $2.533371788483 \mathrm{E}-01$ & -1.40557 & $29646 \mathrm{E}-02$ \\
\hline $\mathrm{S} 2$ & $-2.399202937798 \mathrm{E}-01$ & $2.965857406361 \mathrm{E}-01$ & -4.44157 & $71379 \mathrm{E}-01$ \\
\hline S3 & $-2.600560497510 \mathrm{E}-01$ & $-2.827504872809 \mathrm{E}-01$ & 2.930805 & $9916 \mathrm{E}-01$ \\
\hline $\mathrm{S} 4$ & $4.186724675546 \mathrm{E}-01$ & $-2.553305098232 \mathrm{E}-01$ & 4.030463 & $5229 \mathrm{E}-01$ \\
\hline $\mathrm{O} 1$ & $-1.114524930995 \mathrm{E}-01$ & $-5.850335632794 \mathrm{E}-02$ & -1.93746 & $73700 \mathrm{E}-01$ \\
\hline $\mathrm{O} 2$ & $-3.884457862172 \mathrm{E}-01$ & $4.245957350246 \mathrm{E}-01$ & 4.185051 & $5161 \mathrm{E}-01$ \\
\hline N1 & $-2.167829284962 \mathrm{E}-02$ & $2.763443555973 \mathrm{E}-01$ & -1.77708 & $14048 \mathrm{E}-01$ \\
\hline $\mathrm{N} 2$ & $-1.158456530094 \mathrm{E}-01$ & $2.831946567433 \mathrm{E}-01$ & -3.04358 & $94910 \mathrm{E}-01$ \\
\hline N3 & $-3.828001112086 \mathrm{E}-01$ & $-2.398621921020 \mathrm{E}-01$ & 3.117730 & $4371 \mathrm{E}-01$ \\
\hline N4 & $-4.772788070014 \mathrm{E}-01$ & $-2.370295453277 \mathrm{E}-01$ & 3.437583 & $8708 \mathrm{E}-01$ \\
\hline $\mathrm{C} 1$ & $-8.569092417940 \mathrm{E}-02$ & $1.470696819341 \mathrm{E}-01$ & -2.22172 & $52012 \mathrm{E}-01$ \\
\hline $\mathrm{C} 2$ & $2.229357916710 \mathrm{E}-02$ & $2.568434731523 \mathrm{E}-01$ & -1.05296 & $81037 \mathrm{E}-01$ \\
\hline $\mathrm{C} 3$ & $-1.710067346274 \mathrm{E}-01$ & $2.785778492811 \mathrm{E}-01$ & -3.63835 & $98513 \mathrm{E}-01$ \\
\hline $\mathrm{C} 4$ & $-4.135834538827 \mathrm{E}-01$ & $-3.707120564249 \mathrm{E}-01$ & 3.638099 & $5360 \mathrm{E}-01$ \\
\hline $\mathrm{C} 5$ & $-3.283287821908 \mathrm{E}-01$ & $-2.669550145181 \mathrm{E}-01$ & 3.060725 & $6258 \mathrm{E}-01$ \\
\hline $\mathrm{C} 6$ & $4.785244697367 \mathrm{E}-01$ & $-2.544502460286 \mathrm{E}-01$ & 3.718500 & $1061 \mathrm{E}-01$ \\
\hline
\end{tabular}

Table S4: Band assignments for the calculated Raman spectra $\left(>150 \mathrm{~cm}^{-1}\right)$ of carbonyl diisothiocyanate. The notation for band assignments is the following: $v$-stretching, $\delta$-deformation, s-symmetric, asantisymmetric. The irreducible representations are given for the point groups of the respective space group types of the crystal structures. The observed bands are from measurement with a $532 \mathrm{~nm}$ laser if not mentioned otherwise.

\begin{tabular}{cccc}
$\begin{array}{c}v(\text { calculated }) \\
{\left[\mathrm{cm}^{-1}\right]}\end{array}$ & $\begin{array}{c}v(\text { observed }) \\
{\left[\mathrm{cm}^{-1}\right]}\end{array}$ & irrep. & assignment \\
\hline \hline & & \multicolumn{2}{c}{ Carbonyl diisothiocyanate } \\
\hline 2159 & & $\mathrm{Ag}$ & \\
2144 & not observed & $\mathrm{Ag}$ & $v_{\text {as }}-$ antisymmetric NCS stretching \\
2124 & & $\mathrm{Bg}$ & \\
2120 & & $\mathrm{Bg}$ & \\
\hline 2091 & & $\mathrm{Bg}$ & \\
2087 & & $\mathrm{Bg}$ & \\
2061 & not observed & $\mathrm{Ag}$ & \\
2038 & & $\mathrm{Ag}$ & \\
\hline 1792 & & $\mathrm{Ag}$ & \\
1787 & & $\mathrm{Bg}$ & \\
1786 & 1670 & $\mathrm{Bg}$ & \\
1784 & & $\mathrm{Ag}$ & \\
\hline 1364 & not observed & $\mathrm{Bg}$ & $\delta-$ in plane rocking $\delta\left(\mathrm{O}=\mathrm{CN}_{2}\right)$ deformation
\end{tabular}




\begin{tabular}{|c|c|c|c|}
\hline 1363 & & $\mathrm{Bg}$ & \\
\hline 1335 & & $\mathrm{Ag}$ & \\
\hline 1328 & & $\mathrm{Ag}$ & \\
\hline 1148 & \multirow{4}{*}{1084} & $\mathrm{Ag}$ & \multirow{4}{*}{$\begin{array}{l}v(\mathrm{C}=\mathrm{O}) \text { stretching coupled with } v(\mathrm{C}-\mathrm{N}) \text { stretching and minor } \\
\text { amount of } v_{\mathrm{s}}(\mathrm{NCS}) \text { stretching }\end{array}$} \\
\hline 1146 & & $\mathrm{Ag}$ & \\
\hline 1145 & & $\mathrm{Bg}$ & \\
\hline 1144 & & $\mathrm{Bg}$ & \\
\hline 864 & \multirow{4}{*}{ not observed } & $\mathrm{Bg}$ & \multirow{4}{*}{$\delta$ - in plane rocking $\delta\left(\mathrm{O}=\mathrm{CN}_{2}\right)$ deformation } \\
\hline 863 & & $\mathrm{Bg}$ & \\
\hline 849 & & $\mathrm{Ag}$ & \\
\hline 846 & & $\mathrm{Ag}$ & \\
\hline 809 & \multirow{4}{*}{808} & $\mathrm{Ag}$ & \multirow{4}{*}{$\delta$ - scissoring $\delta(\mathrm{N}-\mathrm{C}-\mathrm{N})$ deformation } \\
\hline 805 & & $\mathrm{Ag}$ & \\
\hline 803 & & $\mathrm{Bg}$ & \\
\hline 799 & & $\mathrm{Bg}$ & \\
\hline 735 & \multirow{4}{*}{ not observed } & $\mathrm{Ag}$ & \multirow{4}{*}{$\delta$ - out of plane rocking $\delta\left(\mathrm{O}=\mathrm{CN}_{2}\right)$ deformation } \\
\hline 734 & & $\mathrm{Bg}$ & \\
\hline 733 & & $\mathrm{Ag}$ & \\
\hline 733 & & $\mathrm{Bg}$ & \\
\hline 663 & \multirow{4}{*}{ not observed } & $\mathrm{Bg}$ & \multirow{4}{*}{$\begin{array}{c}\delta \text { - in plane rocking } \delta\left(\mathrm{O}=\mathrm{CN}_{2}\right) \text { deformation coupled with in } \\
\text { plane } \delta(\mathrm{NCS}) \text { deformation }\end{array}$} \\
\hline 6662 & & $\mathrm{Ag}$ & \\
\hline 659 & & $\mathrm{Bg}$ & \\
\hline 658 & & $\mathrm{Ag}$ & \\
\hline 503 & \multirow{12}{*}{484} & $\mathrm{Bg}$ & \multirow{12}{*}{$\delta$ - out of plane rocking $\delta$ (NCS) deformation } \\
\hline 502 & & $\mathrm{Bg}$ & \\
\hline 502 & & $\mathrm{Ag}$ & \\
\hline 501 & & $\mathrm{Ag}$ & \\
\hline 497 & & $\mathrm{Ag}$ & \\
\hline 495 & & $\mathrm{Ag}$ & \\
\hline 492 & & $\mathrm{Bg}$ & \\
\hline 492 & & $\mathrm{Bg}$ & \\
\hline 489 & & $\mathrm{Bg}$ & \\
\hline 488 & & $\mathrm{Ag}$ & \\
\hline 488 & & $\mathrm{Bg}$ & \\
\hline 486 & & $\mathrm{Ag}$ & \\
\hline 469 & \multirow{4}{*}{ not observed } & $\mathrm{Bg}$ & \multirow{4}{*}{$\delta-$ in plane rocking $\delta$ (NCS) deformation } \\
\hline 468 & & $\mathrm{Ag}$ & \\
\hline 468 & & $\mathrm{Bg}$ & \\
\hline 467 & & $\mathrm{Ag}$ & \\
\hline 331 & \multirow{4}{*}{331} & $\mathrm{Bg}$ & \multirow{4}{*}{$\begin{array}{c}\delta \text { - scissoring } \delta(\mathrm{N}-\mathrm{C}-\mathrm{N}) \text { deformation coupled with minor } \\
\text { amounts of } v(\mathrm{NCS}) \text { stretching }\end{array}$} \\
\hline 331 & & $\mathrm{Ag}$ & \\
\hline 330 & & $\mathrm{Bg}$ & \\
\hline 329 & & $\mathrm{Ag}$ & \\
\hline 143 & \multirow{3}{*}{ not observed } & $\mathrm{Bg}$ & \multirow{3}{*}{$\delta$ - out of plane rocking $\delta\left(\mathrm{O}=\mathrm{CN}_{2}\right)$ deformation } \\
\hline 142 & & $\mathrm{Ag}$ & \\
\hline 139 & & $\mathrm{Ag}$ & \\
\hline
\end{tabular}




\begin{tabular}{cccc}
138 & & $\mathrm{Bg}$ & \\
\hline 131 & & $\mathrm{Ag}$ & \\
129 & $\mathrm{Bg}$ & \\
129 & 131 & $\mathrm{Bg}$ & \\
128 & & $\mathrm{Ag}$ & \\
\hline 101 & & $\mathrm{Ag}$ & \\
98 & 96 & $\mathrm{Bg}$ & \\
97 & & $\mathrm{Ag}$ & \\
94 & & $\mathrm{Ag}$ & \\
74 & & $\mathrm{Bg}$ & \\
72 & & $\mathrm{Ag}$ & \\
72 & not observed & $\mathrm{Bg}$ & \\
63 & & $\mathrm{Ag}$ & \\
\hline 62 & & $\mathrm{Ag}$ & \\
56 & & $\mathrm{Bg}$ & \\
55 & 50 & $\mathrm{Bg}$ & \\
51 & & $\mathrm{Ag}$ & \\
\hline
\end{tabular}


Table S5: Lattice parameters and atomic coordinates of the optimized solid-state structure of oxalyl diisothiocyanate.

Lattice parameter $a$ $b$ 5.35080025 20.55573998 5.91228121

\begin{tabular}{llll} 
Atom & \multicolumn{1}{c}{$x$} & \multicolumn{1}{c}{$y$} & \multicolumn{1}{c}{$z$} \\
\hline \hline C1 & $1.202023776714 \mathrm{E}-01$ & $1.160303126324 \mathrm{E}-02$ & $6.052973712065 \mathrm{E}-02$ \\
C2 & $6.427148216656 \mathrm{E}-03$ & $1.241252206276 \mathrm{E}-01$ & $3.926091172470 \mathrm{E}-02$ \\
N1 & $1.319480718109 \mathrm{E}-01$ & $7.744467913842 \mathrm{E}-02$ & $8.985353724423 \mathrm{E}-02$ \\
O1 & $2.770877770646 \mathrm{E}-01$ & $-2.592874331598 \mathrm{E}-02$ & $1.217147856285 \mathrm{E}-01$ \\
S1 & $-1.303001156292 \mathrm{E}-01$ & $1.892714745838 \mathrm{E}-01$ & $-1.113016128157 \mathrm{E}-02$ \\
\hline \hline
\end{tabular}

Table S6: Band assignments for the calculated Raman spectra $\left(>150 \mathrm{~cm}^{-1}\right)$ of carbonyl diisothiocyanate and oxalyl diisothiocyanate. The notation for band assignments is the following: $v$-stretching, $\delta-$ deformation, s-symmetric, as-antisymmetric. The irreducible representations are given for the point groups of the respective space group types of the crystal. The observed bands are from measurement with a $532 \mathrm{~nm}$ laser if not mentioned otherwise.

\begin{tabular}{|c|c|c|c|}
\hline $\begin{array}{c}v(\text { calculated }) \\
{\left[\mathrm{cm}^{-1}\right]}\end{array}$ & $\begin{array}{l}v \text { (observed) } \\
{\left[\mathrm{cm}^{-1}\right]}\end{array}$ & irrep. & assignment \\
\hline \multicolumn{4}{|r|}{ Oxalyl diisothiocyanate } \\
\hline 2135 & \multirow{2}{*}{ not observed } & B1g & \multirow{2}{*}{$v_{\text {as }}-$ antisymmetric NCS stretching } \\
\hline 2134 & & $\mathrm{Ag}$ & \\
\hline 2045 & \multirow{2}{*}{ not observed } & B3g & \multirow{2}{*}{$\begin{array}{c}v_{\text {as }}-\text { antisymmetric NCS stretching coupled with } v(\mathrm{C}=\mathrm{O}) \\
\text { stretching }\end{array}$} \\
\hline 2045 & & $\mathrm{~B} 2 \mathrm{~g}$ & \\
\hline 1848 & \multirow{2}{*}{1742} & B1g & \multirow{2}{*}{$v(\mathrm{C}=\mathrm{O})$ stretching coupled with $v(\mathrm{C}-\mathrm{C})$ stretching } \\
\hline 1848 & & $\mathrm{Ag}$ & \\
\hline 1793 & \multirow{2}{*}{1688 (weak) } & B2g & \multirow{2}{*}{$\begin{array}{c}v(\mathrm{C}=\mathrm{O}) \text { stretching coupled with } v(\mathrm{C}-\mathrm{C}) \text { stretching and minor } \\
\text { amount of } v_{\text {as }}(\mathrm{NCS}) \text { stretching }\end{array}$} \\
\hline 1792 & & B3g & \\
\hline 1321 & \multirow{4}{*}{ not observed } & $\mathrm{Ag}$ & \multirow{4}{*}{$v(\mathrm{C}-\mathrm{N})$ stretching coupled with symmetric NCS stretching } \\
\hline 1319 & & B1g & \\
\hline 1305 & & B3g & \\
\hline 1304 & & $\mathrm{~B} 2 \mathrm{~g}$ & \\
\hline 1030 & \multirow{4}{*}{999} & $\mathrm{Ag}$ & \multirow{4}{*}{$v(\mathrm{C}-\mathrm{C})$ coupled with $v_{\mathrm{s}}(\mathrm{NCS})$ and $\delta(\mathrm{N}-\mathrm{C}-\mathrm{O})$ scissoring } \\
\hline 1030 & & B1g & \\
\hline 1026 & & B3g & \\
\hline 1025 & & $\mathrm{~B} 2 \mathrm{~g}$ & \\
\hline 783 & \multirow{4}{*}{ not observed } & B3g & \multirow{4}{*}{$\delta$ - out of plane $\delta(\mathrm{C}-\mathrm{C})$ deformation } \\
\hline 783 & & $\mathrm{~B} 2 \mathrm{~g}$ & \\
\hline 769 & & B1g & \\
\hline 769 & & $\mathrm{Ag}$ & \\
\hline 677 & \multirow{4}{*}{655} & B3g & \multirow{4}{*}{$\begin{array}{c}\delta-(\mathrm{O}=\mathrm{C}-\mathrm{C}=\mathrm{O}) \text { symmetric stretching coupled with NCS } \\
\text { scissoring }\end{array}$} \\
\hline 677 & & $\mathrm{~B} 2 \mathrm{~g}$ & \\
\hline 674 & & B1g & \\
\hline 674 & & $\mathrm{Ag}$ & \\
\hline 599 & not observed & B3g & \\
\hline
\end{tabular}




\begin{tabular}{|c|c|c|c|}
\hline 599 & & $\mathrm{Ag}$ & \multirow{3}{*}{$\begin{array}{c}\delta-(\mathrm{O}=\mathrm{C}-\mathrm{C}=\mathrm{O}) \text { antisymmetric stretching coupled with NCS } \\
\text { scissoring }\end{array}$} \\
\hline 598 & & B2 $g$ & \\
\hline 598 & & B1g & \\
\hline 495 & \multirow{4}{*}{ not observed } & B1g & \multirow{4}{*}{$\delta$ - out of plane (NCS) deformation } \\
\hline 489 & & $\mathrm{Ag}$ & \\
\hline 488 & & B2g & \\
\hline 487 & & B3g & \\
\hline 438 & \multirow{4}{*}{422} & B3g & \multirow{4}{*}{ 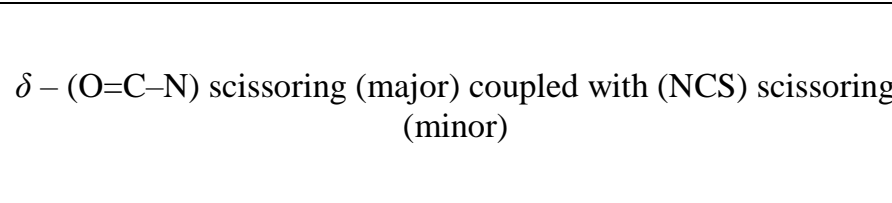 } \\
\hline 437 & & B2g & \\
\hline 434 & & B1g & \\
\hline 433 & & $\mathrm{Ag}$ & \\
\hline 382 & \multirow{4}{*}{373} & B2g & \multirow{4}{*}{$\delta-(\mathrm{O}=\mathrm{C}-\mathrm{N})$ scissoring $\underset{(\text { major) }}{\text { minor) coupled with NCS scissoring }}$} \\
\hline 380 & & B3g & \\
\hline 380 & & B1g & \\
\hline 376 & & $\mathrm{Ag}$ & \\
\hline 191 & \multirow{2}{*}{ not observed } & B2g & \multirow{2}{*}{$\delta-(\mathrm{O}=\mathrm{C}-\mathrm{C}=\mathrm{O})$ wagging } \\
\hline 191 & & B3g & \\
\hline 169 & \multirow{2}{*}{ not observed } & B1g & \multirow{2}{*}{$\begin{array}{c}\delta-\text { out of plane deformation with three nodes over the whole } \\
\text { molecule }\end{array}$} \\
\hline 168 & & $\mathrm{Ag}$ & \\
\hline 129 (shoulder) & \multirow{2}{*}{$121^{[\mathrm{a}]}$} & B3g & \multirow{2}{*}{ lattice vibration } \\
\hline 129 (shoulder) & & B2g & \\
\hline 121 & \multirow{4}{*}{$121^{[\mathrm{a}]}$} & $\mathrm{Ag}$ & \multirow{4}{*}{ lattice vibration } \\
\hline 118 & & B2g & \\
\hline 113 & & B3g & \\
\hline 112 & & B1g & \\
\hline 94 & \multirow{4}{*}{$92^{[\mathrm{a}]}$} & B1g & \multirow{4}{*}{ lattice vibration } \\
\hline 93 & & $\mathrm{Ag}$ & \\
\hline 88 & & B1g & \\
\hline 87 & & B3g & \\
\hline
\end{tabular}

[a] Those bands have only been observed by excitation with a $633 \mathrm{~nm}$ laser. 